\title{
Doppler radar wind field retrieval over the Po Valley
}

\author{
Y. K. Goh ${ }^{1,2}$, A. R. Holt ${ }^{1}$, and P. P. Alberoni ${ }^{3}$ \\ ${ }^{1}$ Department of Mathematical Sciences, University of Essex, Colchester CO4 3SQ, UK \\ ${ }^{2}$ Faculty of Information and Communication Technology, Universiti Tunku Abdul Rahman 13 Jalan 13/6, 46200 Petaling \\ Jaya, Selangor, Malaysia \\ ${ }^{3}$ ARPA-SIM, Viale Silvani 6, 40122, Bologna, Italy
}

Received: 18 October 2005 - Revised: 21 February 2006 - Accepted: 21 February 2006 - Published: 4 May 2006

\begin{abstract}
Although methods of using multiple Doppler radars to study wind fields have long been proposed, and many research studies have been made, very few operational radar operators adopt methods which require the use of specific scanning strategies to allow the extraction of wind information. Here we report a collaborative study on dualDoppler radars based on two Doppler radars in the Po valley, Italy. Unusually, the radars are only about $90 \mathrm{~km}$ apart, though operated by the same authority. The wind field syntheses are carried out on a $30 \mathrm{~km}$ by $30 \mathrm{~km}$ region where the two radars have overlapping scan coverage. An iterative method based on the linear wind model and the equation of mass continuity is used to construct the wind fields. The methodology has been validated by two different methods. The first method is to reconstruct the radial wind observed by each radar, and the second method is calculating and comparing the along-track component with that derived from the observations. Both two comparisons show good agreement with the original data.
\end{abstract}

\section{Introduction}

Doppler weather radars are very important instruments in precipitation nowcasting, and can provide important input to numerical forecasting models. Much of the precipitation estimations used in weather forecasting rely on the reflectivity measurements of weather radars. Doppler radars not only can provide the necessary reflectivity data but also measure the radial Doppler velocities of the hydrometeors in the atmosphere. The Doppler velocity data provide valuable information on the speed of the hydrometeors, and thus the wind speed (by assuming the hydrometeors move with the wind), for use in numerical weather prediction. Although

Correspondence to: A. R. Holt

(anthony@essex.ac.uk) a Doppler radar can provide high resolution information on the wind speed over a wide coverage area, it also suffers from several draw-backs. The first draw-back is the so-called velocity ambiguity dilemma where one has to compromise unambiguous velocity resolution for a longer scanning range or vice-versa. The second draw-back is that Doppler measurements only provide one out of three components of the wind field. Various techniques (see Shapiro et al., 1995; Laroche and Zawadzki, 1994; Lhermitte, 1970) have been developed to address these draw-backs; multiple Doppler wind retrieval is one of the techniques.

The studies presented in this paper are part of the Work Package 2 of the EU Carpe Diem project. At present, many operational weather radars in Europe are capable of measuring Doppler wind data. The objective of Work Package 2 (WP2) of the Carpe Diem project was to increase the exploitation of the Doppler data by two different strategies: (1) direct extraction of wind information from Doppler data, and (2) Generation of a superobservation product from the Doppler data and its incorporation into a numerical weather prediction (NWP) model (Lindskog et al., 2004). This paper is concerned with the direct wind information extraction. The study of the superobservation product and the integration of the wind speed into numerical weather prediction model has been reported by Haase and Landelius (2004).

There has been one previous attempt to use data from two European weather service radars to derive horizontal wind fields. This was a study using the radars at San Pietro Capofiume and Teolo by Watson (1996). However, the study was hampered by the fact that the radars were not synchronized, and by the topographical factors.

In this study, we use data from two Doppler radars operated by the same weather service, with synchronised scanning strategy, to derive the wind field. Both are located in a flat terrain, which makes some low level observations easy. We demonstrate that one component of the wind field can be derived without the use of the continuity equation. We

Published by Copernicus GmbH on behalf of the European Geosciences Union. 


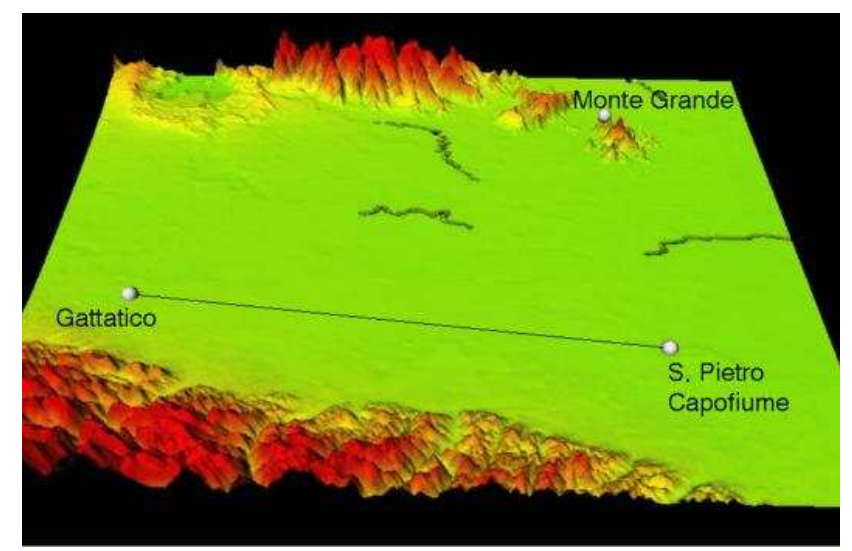

Fig. 1. Terrain topography of the region close to the radars. Two radars in the lower part of the figure are the GAT (Gattatico) and the SPC (San Pietro Capofiume) radars. The distance between the two radars, that represented by the straight line, is approximately $89 \mathrm{~km}$. The third radar, TEO, is located at a higher altitude on the mountain of Monte Grande.

show that it is in good agreement with the component of the calculated wind field vector, thus providing a partial verification of the retrieval. We give examples in both stratiform and convective conditions.

\section{Po Valley, Italy}

The eastern Po valley region can experience severe convective storms and this region has one of the best weather radar coverage in Europe. The two radars used in this study belong to ARPA-SIM (previously known as SMR), the Civil Defence Agency of the Emilia-Romagna region. One is located at San Pietro Capofiume, SPC $\left(44^{\circ} 39^{\prime} 17^{\prime \prime} \mathrm{N}, 11^{\circ} 37^{\prime} 25^{\prime \prime} \mathrm{E}\right)$ and the other at Gattatico, GAT $\left(44^{\circ} 47^{\prime} 30^{\prime \prime} \mathrm{N}, 10^{\circ} 30^{\prime} 30^{\prime \prime} \mathrm{E}\right)$. Both the GAT and the SPC radars are synchronised and employ the same scanning strategy. The set of scans is repeated every $15 \mathrm{~min}$, and consists of 3 different volume scans: (1) $4 \mathrm{~min}$ and 4 different elevation angles dual PRF scan, (2) $8 \mathrm{~min}$ and 15 different elevation angles single PRF scan, and (3) $3 \mathrm{~min}$ and 5 different elevation angles long range (nonDoppler) scan. We have used mainly the dual PRF Doppler Data to perform the wind retrieval to avoid the aliasing problem, since the unambiguous velocity range is $-49 \mathrm{~m} / \mathrm{s}$ to $+49 \mathrm{~m} / \mathrm{s}$. However, the analysis could be extended to single PRF scans if the proper pre-processing has been carried out (see Bergen and Albers, 1988; Haase and Landelius, 2004). The gate size of the radar beams for both ARPA-SIM radars varies for different volume scans. It is $250 \mathrm{~m}$ in dual PRF scan mode, $125 \mathrm{~m}$ for the single PRF scan and $1000 \mathrm{~m}$ in the long range scan.

The terrain height in the region of the radar sites is an important factor in determining the area for performing multiple-Doppler wind retrieval. A suitable area for
multiple-Doppler wind retrieval would be an area with overlapping coverage from all the participating radars and free from beam blockage. The retrieval area should be away from the straight line connecting any two radars otherwise both radars would essentially be reading the same information, that is the same wind component but in the opposite directions. The altitude difference of different radars is crucial for determining the lowest elevation angle that can be used in the retrieval process. The terrain also influences the choice of boundary condition to be used in dual-Doppler analysis. A flat terrain allows the use of a lower boundary condition (Chong et al., 2000; Wüest et al., 1999) in dualDoppler analysis since the vertical velocity on the ground can be assumed to be zero. On the other hand, in a mountainous terrain, one has to resort to using the atmospheric boundary layer as an upper boundary condition (Watson, 1996). The process of identifying the boundary layer usually is not straight forward.

From the map in Fig. 1, the ideal retrieval area will be the flat plane to the north of the line connecting the GAT and SPC radars. The area to the south of the line connecting the GAT and SPC radars is not ideal for the analysis as no low elevation measurement could be taken without the influence of clutter and it would be too remote from another third radar. The possible useful third radar is from the ARPAVeneto Teolo, TEO $\left(45^{\circ} 21^{\prime} 46^{\prime \prime} \mathrm{N}, 11^{\circ} 40^{\prime} 25^{\prime \prime} \mathrm{E}\right)$ radar which is located near Monte Grande. The TEO radar, together with GAT and SPC, form a triangle with sides approximately $112 \mathrm{~km}, 89 \mathrm{~km}$, and $80 \mathrm{~km}$. The TEO radar is potentially useful for the purpose of checking dual Doppler wind fields that were generated by GAT and SPC radar data, and possibly performing triple Doppler analysis. Unfortunately, to date, we have not had sufficient data for these purposes, and this work is left for a future publication.

\section{Data gridding}

The raw data from the ARPA-SIM radars is measured in polar coordinates. It is usually easier to convert the raw data into Cartesian coordinates when performing dual Doppler wind analysis. We used a simple algorithm to convert the data: the chosen area is divided into rectangular grid, with each cell in the grid having dimension of $0.5 \mathrm{~km} \times 0.5 \mathrm{~km}$. Then, individual pixels (bins) from the radar data which fall inside the grid cell are identified. Each Cartesian cell is assigned the value of the bin falling into that particular cell. If more than one bin fall into the same cell, the value of cell is the average of those of the bins. Figure 2 is an example of the data conversion, it shows that the conversion retains most of the features of the orginal data. 


\section{Wind extraction}

Dual Doppler wind retrieval is achieved by solving the standard linear wind field model coupled with the equation of mass continuity

$$
\begin{aligned}
\hat{\boldsymbol{r}}_{\boldsymbol{i}} \cdot \boldsymbol{v} & =u \sin \alpha_{i} \cos \phi_{i}+v \cos \alpha_{i} \cos \phi_{i}+w \sin \phi_{i} \\
& =v_{r_{i}}-\sin \phi_{i} W_{t} \equiv V_{i}, \quad i=1,2 \\
\frac{\partial \rho}{\partial t}+ & \nabla \cdot(\rho \boldsymbol{v})=0,
\end{aligned}
$$

where, $\quad \hat{\boldsymbol{r}}_{\boldsymbol{i}}=\sin \alpha_{i} \cos \phi_{i} \boldsymbol{i}+\cos \alpha_{i} \cos \phi_{i} \boldsymbol{j}+\sin \phi_{i} \boldsymbol{k}$ is the unit vector of the direction of ray from the $i$-th radar; $\boldsymbol{v}(\boldsymbol{r})=(u, v, w)$ is the wind vector at the location $\boldsymbol{r}$ with $u$, $v$ and $w$ as the three Cartesian components; $\alpha$ is the azimuth angle clockwise from the north and $\phi$ is the elevation angle; $v_{r_{i}}$ is the Doppler velocity measured by $i$-th radar; $V_{i}$ is the component of the wind vector towards the radar; and $W_{t}$ is the terminal fall velocity of the scatterers.

We have used an iterative method to solve the equations and the method will be outlined here. A more detailed discussion of the method can be found in Dowell and Shapiro (2003), and Goh and Holt (2004). First, Eq. (1) is rearranged so that now the horizontal wind components are linear functions of the vertical wind component.

$u=A+B w$,

and

$v=C+D w$.

The parameters $A, B, C$ and $D$ (Dowell and Shapiro, 2003) depend on the azimuth angle, elevation angle and radial Doppler wind measured from radars. Then, Eq. (2) is discretised to give

$$
\begin{aligned}
\left(1+\frac{\Gamma \Delta z}{2}\right) w_{k}= & \left(1-\frac{\Gamma \Delta z}{2}\right) w_{k-1} \\
& -\left(\frac{\partial u}{\partial x}+\frac{\partial v}{\partial y}\right) \Delta z,
\end{aligned}
$$

where the subscript $k$ indicates the number of iterations, and $\Gamma \equiv \partial(\ln \rho) / \partial z$ is the logarithmic vertical pressure variation, and it is set to $-0.0001 \mathrm{~m}^{-1}$ (Dowell and Shapiro, 2003).

The boundary conditions used in our calculation assumes the vertical component of the wind on the ground to be zero. Then, Eq. (2) is integrated from bottom to top. The reasons we use the lower boundary condition are threefold. Firstly, we would like to use the dual PRF scans which have the Nyquist velocity of $49 \mathrm{~m} / \mathrm{s}$ to avoid aliasing problem, and the dual PRF scans usually are lower than the atmospheric boundary layer. Secondly, obtaining the upper boundary layer is not always straight forward. Thirdly, usually the wind is stronger higher in the atmosphere, and integration from a strong wind higher atmospheric region to weak wind lower atmospheric region is often problematic.
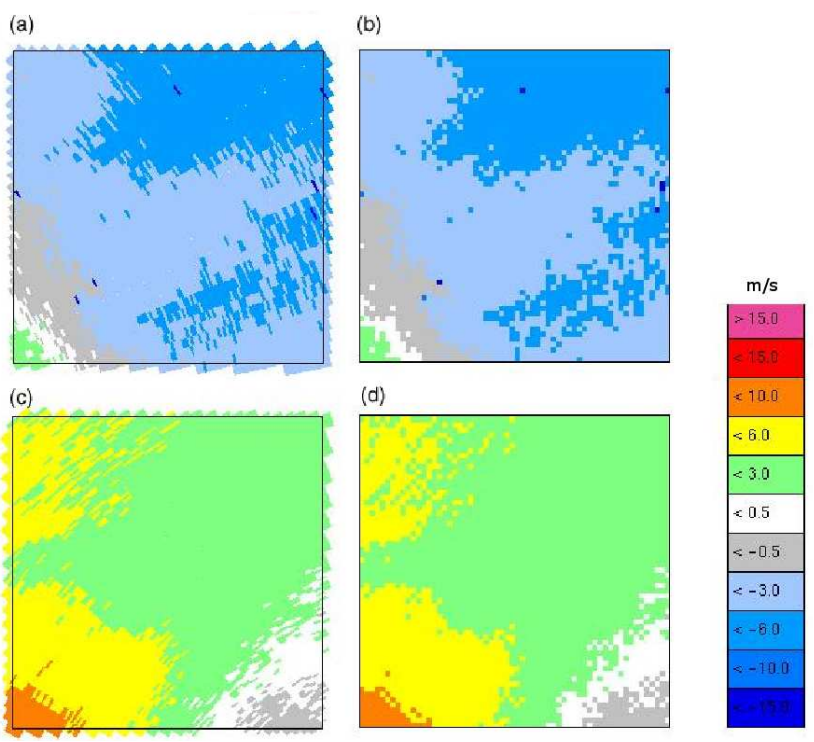

Fig. 2. Example of the output from the data gridding algorithm. (a) and (c) show the section of PPI displays of the raw Doppler radial velocity data in the retrieval area. (b) and (d) show the same PPI sections after applying the gridding procedure .

We have used the raw data from the both SPC and GAT radars to construct the wind field. The data was obtained from dual PRF scans which reduced the aliasing ambiguity problem. We have gridded the data into 4 horizontal layers with each layer separated by $0.9 \mathrm{~km}$ vertical spacing. Each layer is gridded into $0.5 \times 0.5 \mathrm{~km}^{2}$. Hence the resulting wind field is a three-dimensional wind field on a $0.5 \times 0.5 \times 0.9 \mathrm{~km}^{3}$ grid. Some of the retrieved wind fields are shown in the following section.

\section{Dual Doppler wind fields}

We now show some examples of the wind fields retrieved by our method. Fig. 3 shows the Doppler velocity PPI at SPC on 9 September 2003 at a $1.4^{\circ}$ elevation angle. For both radars, a positive Doppler radial velocity means that the wind component is directed towards the radar. The square box in Fig. 3 is the chosen area for dual Doppler wind retrieval.

A common practice in extracting wind information from Doppler data is to use a Velocity Azimuth Diagram, VAD (Lhermitte, 1970). A low-elevation VAD diagram will look like a sine curve if the wind field is uniform. However, the VAD method can be difficult to interpret for a non-uniform wind field condition, such as in our case here. The VAD diagrams for both SPC and GAT radars are shown in Fig. 4. Both VAD diagrams have the same range $(58 \mathrm{~km})$ which is approximately equal to the distances from the radars to the centre of the box. The erratic VAD curves sugguest that the wind field is not uniform in this region. 


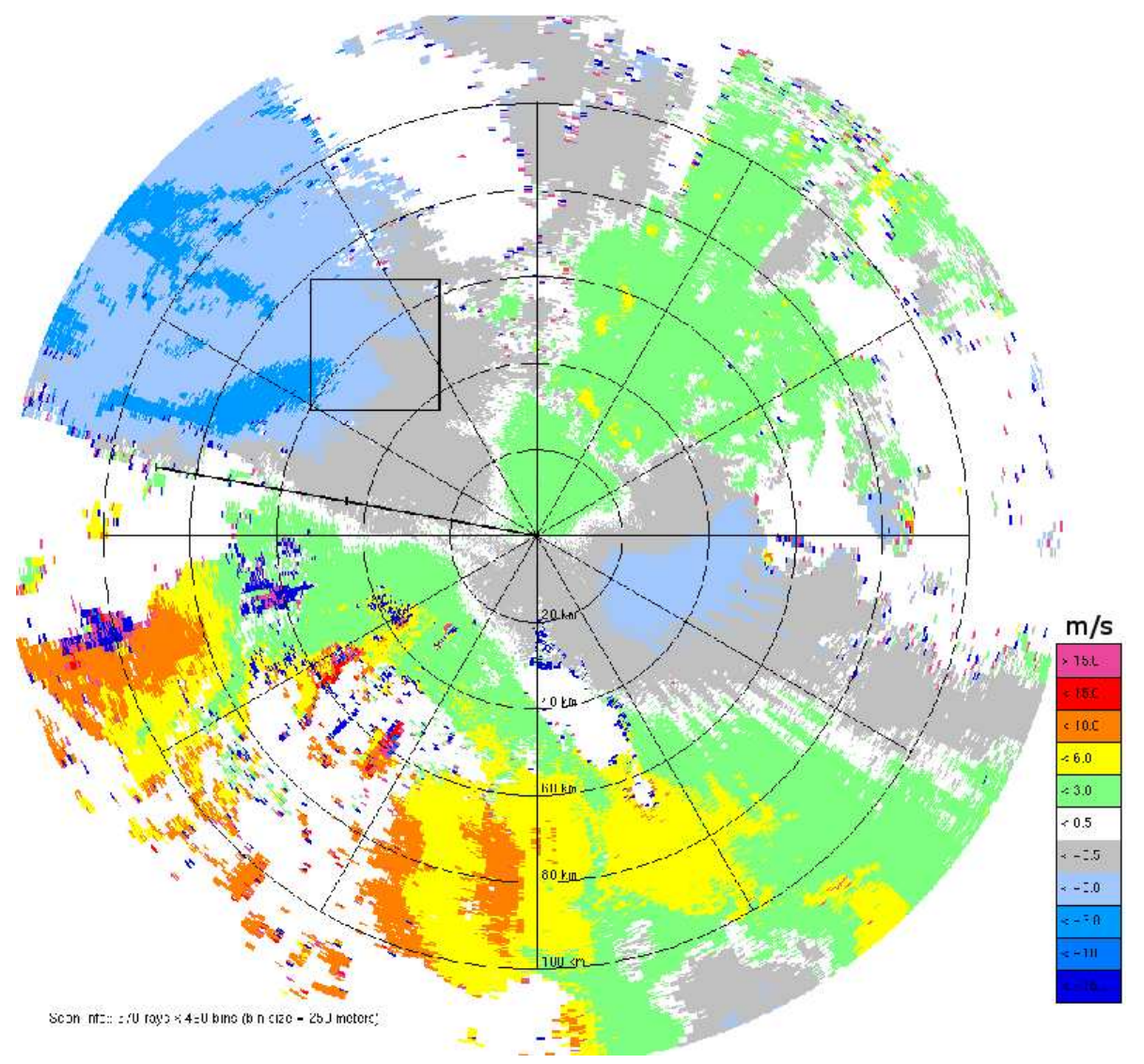

Fig. 3. Doppler velocity PPI picture of SPC radar at the elevation angle of $1.4^{\circ}$ ( 9 September 2003, 13:15 h). The two ends of the straight line are the locations of the two radars used for dual-Doppler wind analysis. The retrieval area is shown by the square box.

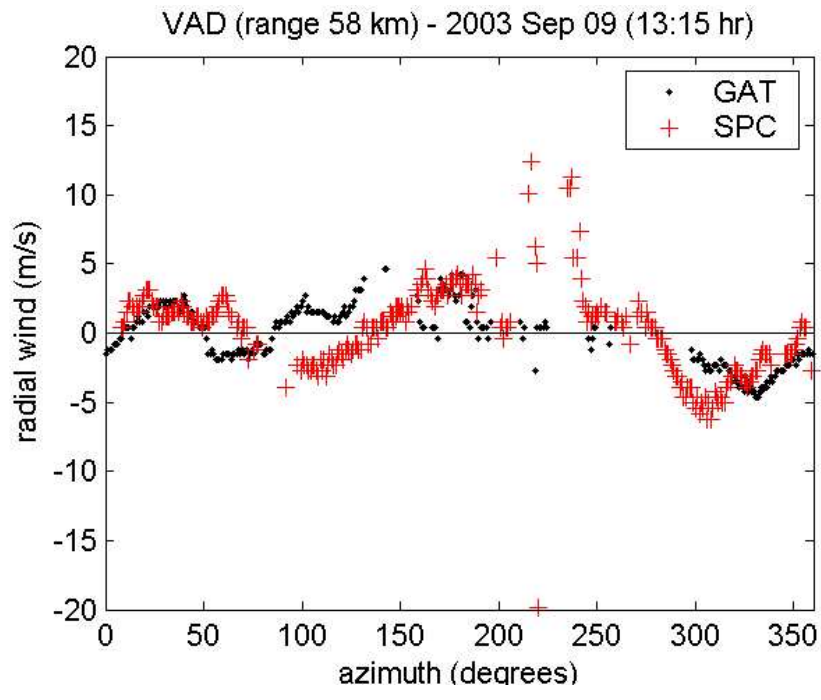

Fig. 4. Figure shows the VAD diagram of the GAT and SPC radars (9 September 2003, 13:15 h, elevation angle $1.4^{\circ}$ ). The VAD curves are far from being similar to sine curves, which shows that the windfield is not uniform.
Dual Doppler wind retrieval does not rely on the uniform wind field assumption and it can be very useful in this situation. Figure 5 shows the horizontal wind field extracted from the ARPA-SIM radar dataset by a dual Doppler wind analysis. The extracted wind field is three-dimensional, Fig. 5 only shows the horizontal cross section at a height of $2.3 \mathrm{~km}$. Another horizontal cross section of the wind field at different height is shown in Fig. 6. We can clearly see the wind is in a westerly direction at the south of the retrieval area and eventually turns to a north-west direction in the north. The inserts in Fig. 5 are the PPIs from the gridded Doppler velocity data in the retrieval area.

Figures 6 and 7 show the vertical as well as the horizontal cross sections of the wind field constructed from dualDoppler wind analysis for two different days. From Fig. 6, the vertical movement of the hydrometeors is not obvious. The general trend of the wind motion is directed towards the North. On the other hand, Fig. 7 shows a convective case. The horizontal cross section shows that the convective circulation at the lower centre part of the figure. The corresponding vertical cross section of the wind field shows an active up draft motion in the centre of the figure. For the stratiform part, the wind field is generally moving to the South. 

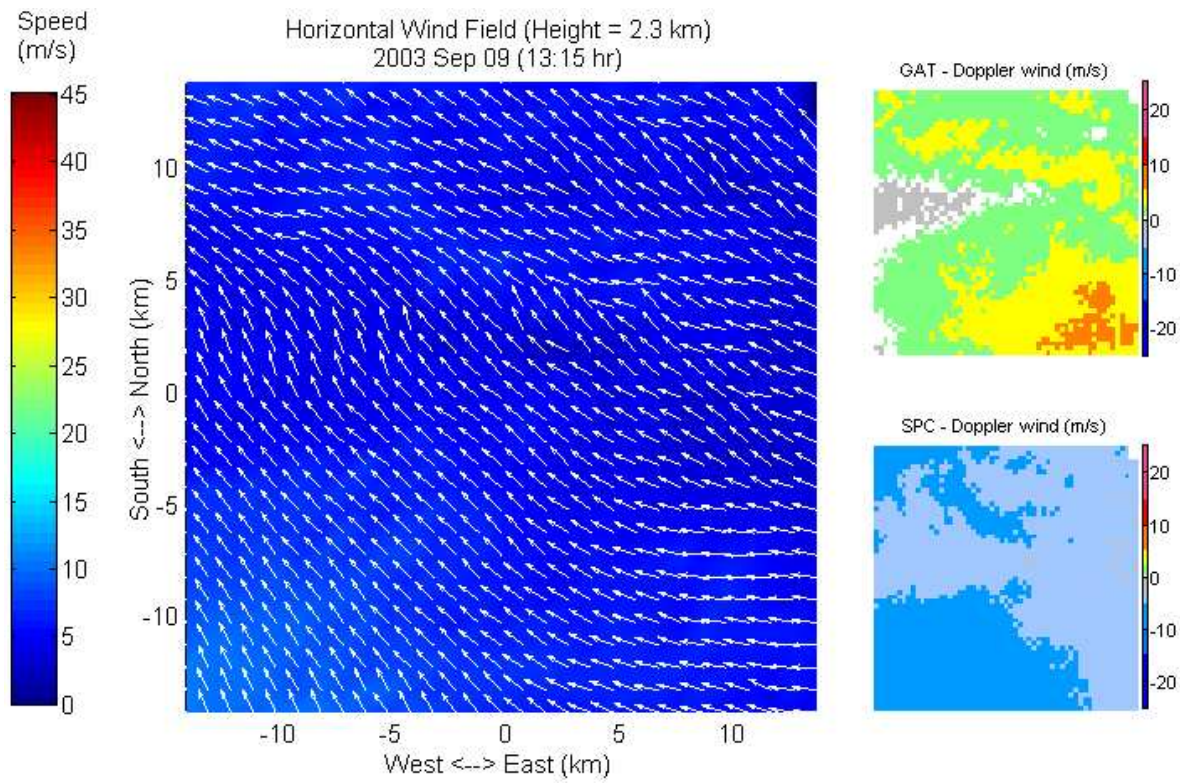

Fig. 5. Horizontal cross section of the wind field constructed from dual-Doppler wind analysis at one height (9 September 2003, 13:15 h). The centre vector field represents the wind field direction and the back ground colour represents the magnitude of the wind speed. The two small figures on right hand side are the gridded Doppler wind data from GAT and SPC.

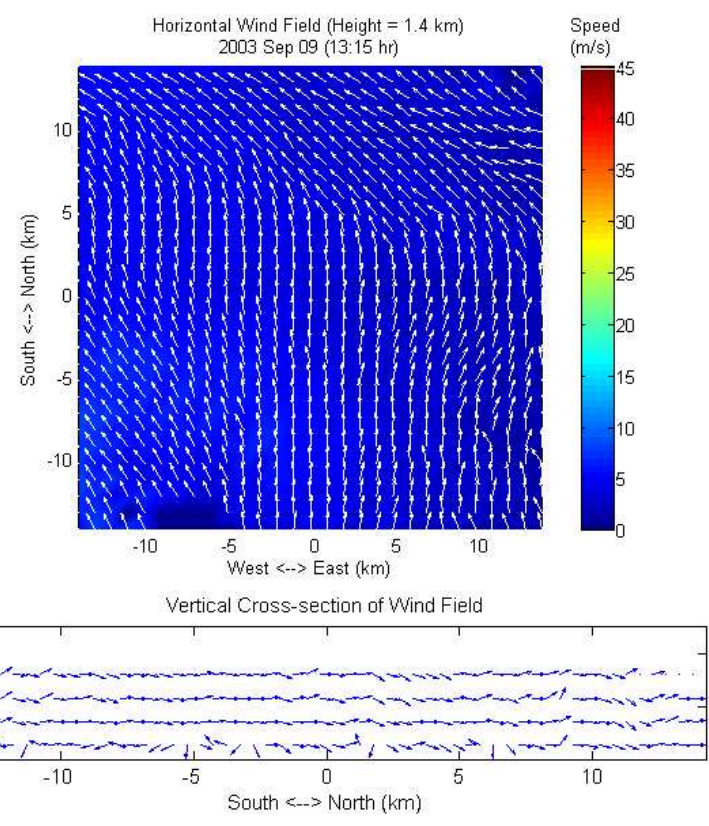

Fig. 6. Horizontal and vertical wind field cross sections constructed from dual-Doppler wind analysis (9 September 2003, 13:15 h) The horizontal cross section (top) is taken at a height of $1.4 \mathrm{~km}$. The vertical cross section (bottom) is taken across the North-south direction and passes through the centre of the retrieval area. The field arrows in the figures are normalised to unity for clarity. It is clear that the wind field is stratiform since there is only slight vertical movement.
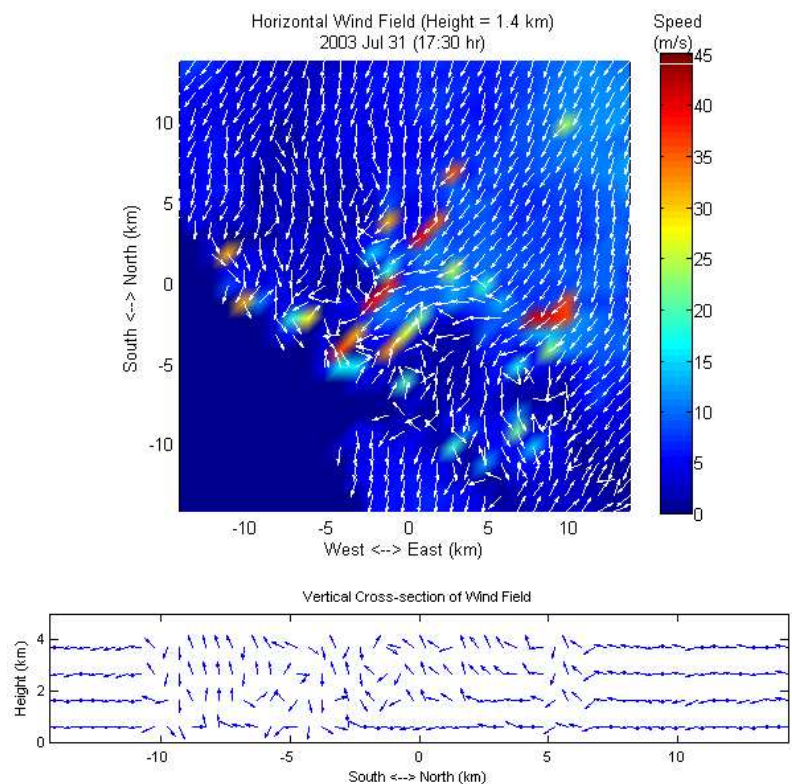

Fig. 7. Horizontal and vertical wind field cross sections constructed from dual-Doppler wind analysis. The date of the radar data is 31 July 2003 at 17:30 h. The horizontal cross section (top) is taken at a height of $1.4 \mathrm{~km}$. The vertical cross section (bottom) is taken across the North-south direction and passes through the centre of the retrieval area. The field arrows in the figures are normalised to unity for clarity. The vertical cross section of the wind field shows active vertical up draft in the region between $-10 \mathrm{~km}$ to $5 \mathrm{~km}$, while the horizontal cross section shows that the wind field is a convective wind field. 
(a)

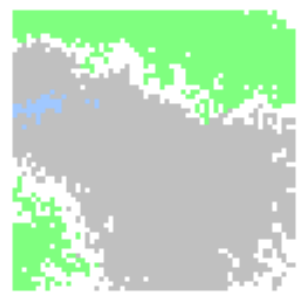

(c)

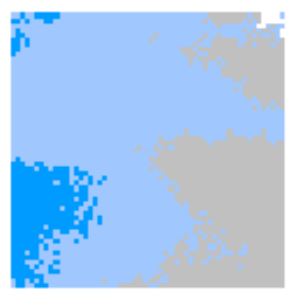

(b)

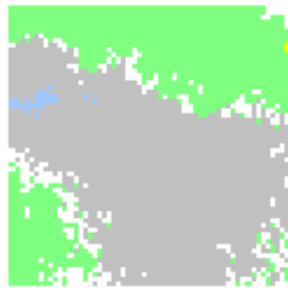

(d)

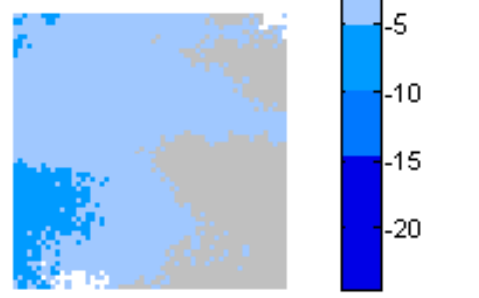

Fig. 8. Figures show the comparison of PPI pictures using reconstruction method. (a) PPI showing the gridded data of the GAT radar. (b) PPI reconstructed from dual Doppler wind-field as would be seen from the GAT radar. (c) PPI showing the gridded data of the SPC radar. (d) PPI reconstructed from dual Doppler wind-field as would be seen from the SPC radar. The data was taken at 13:15 $\mathrm{h}$ on 9 September 2003.

Once we have constructed the wind field, the next task is to validate it. Since there is no independent measurement of the wind velocity vector in the same region, we have to make use of the available radar data to verify the extracted wind-field. We used two different validation methods. First, after the wind-field is extracted, we can re-calculate the radial component of the wind vector as seen from the radar by performing a vector projection and comparing the result with the Doppler wind that was measured by the radar. Another method of validating the wind field is by calculating the along-track component of the wind vector.

Figure 8 shows the comparison of the gridded PPI data and the reconstructed PPIs of the radial winds toward the radars. Figure $8 \mathrm{a}$ is the gridded PPI data for GAT radar and Fig. 8b is the corresponding reconstructed PPI. Similarily, Figs. 8c and $d$ are the corresponding gridded PPI and reconstructed PPI for SPC radar. We can see there is excellent agreement between the gridded data and the recontructed picture.

\section{Along-track (AT) components method}

It is always possible to unambiguously determine the wind component along the line connecting two Doppler radars. We have called this the along-track (AT) component. This alongtrack component of the wind vector can be used to compare with the wind field extracted from dual-Doppler analysis and provide us with a measure of how well it fits the radar measurements.

To obtain the along-track component, let us assume that both radars are looking at the same point and the displacements vectors from the radars are denoted by $\boldsymbol{r}_{\mathbf{1}}$ and $\boldsymbol{r}_{\mathbf{2}}$, and the displacement from first radar to second radar is $\boldsymbol{r}_{12}=\boldsymbol{r}_{1}-\boldsymbol{r}_{2}$. Thus,

$\boldsymbol{v} \cdot \boldsymbol{r}_{12}=V_{1}\left|\boldsymbol{r}_{1}\right|-V_{2}\left|\boldsymbol{r}_{2}\right|$.

The the radial velocity terms on right hand side of Eq. (6) can be obtained from the two Doppler radar measurement. On the other hand, we could use the wind vector derived from dual-Doppler analysis to calculate the left hand side expression. The difference between the left hand side and right hand side of the equation can be used as a indicator of how well the extracted wind field fit the data. Thus we defined the relative AT deviation from observation as

$\frac{\Delta}{|v|\left|r_{12}\right|}=\frac{\boldsymbol{v} \cdot \boldsymbol{r}_{\mathbf{1 2}}-V_{1}\left|\boldsymbol{r}_{\mathbf{1}}\right|+V_{2}\left|\boldsymbol{r}_{\mathbf{2}}\right|}{|v|\left|r_{12}\right|}$.

Figure 9a shows an example of the magnitude of the relative difference between the along-track component derived from radar data (Fig. 9b) and the along-track component calculated from the wind field obtained from dual-Doppler analysis (Fig. 9c). From the colour scale, the maximum relative difference is estimated to be approximately $1 \%$.

\section{Conclusions}

We have shown that it is possible to extract useful wind information from two spatially separated operational Doppler radars. We have successfully analysed many datasets from ARPA-SIM, by using the normal VAD technique, by our dual-Doppler analysis, and by our newly developed alongtrack method. Our research has shown that we can obtain a better description of the wind field in the limited study area than is provided by the VAD method. For example we have been able to derive not only the horizontal component of the wind, but also its component in a vertical plane. Detailed wind field information should be very beneficial both for nowcasting in convective storms, and for research in atmospheric processes and forecasting.

The validation of the analysis methodology has been rigorous. We have used different procedures to check the derived wind fields and all indicate that the outcomes are indeed accurate. We checked the convergence factor in our iterative algorithm to ensure that there is no accumulation of errors. We predicted and reconstructed PPI pictures from the extracted wind-field, and then compared with observations. We also found that the deviation of the along-track component of extracted wind field from the observation is negligible. 


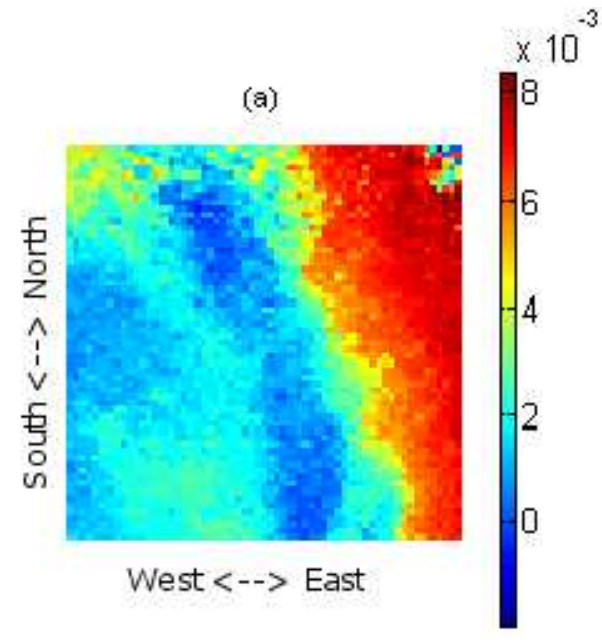

(b)
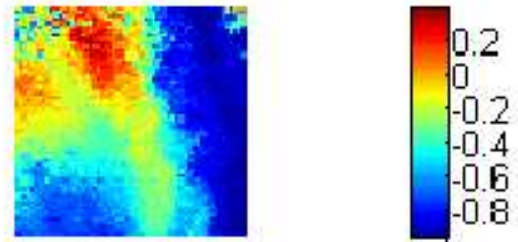

(c)
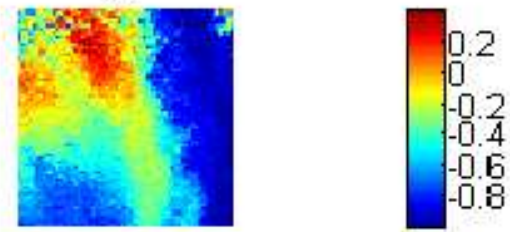

Fig. 9. Figures show the along-track validation method. (a) The relative difference of the along-track component derived from two different methods. (b) Normalised along-track component calculated from gridded data. (c) Normalised along-track component calculated from the extracted wind field. Note that all quantities here are dimensionless quantities.

Acknowledgements. The authors would like to thank the European Commission for supporting the CARPE DIEM project (contract no EVG1-CT-2001-0045). We thank D. Thompson (UK Met Office) for a suggestion which led to the development of the along-track method

Edited by: M. Bruen

Reviewed by: M. Wüest and another referee

\section{References}

Bergen, W. R. and Albers, S. C.: Two- and three-dimensional dealiasing of Doppler radar velocities, J. Atmos. Ocean Technol., 5, 305-319, 1988.

Chong, M., Georgis, J.-F., Bousquet, O., Brodzik, S. R., Burghart, C., Cosma, S., Germann, U., Gouget, V., Houze Jr., R. A., James, C. N., Prieur, S., Rotunno, R., Roux, F., Vivekanandan, J., and Zeng, Z.-X.: Real-time wind synthesis from Doppler radar observations during the Mesoscale Alpine Programme, Bull. Amer. Meteorol. Soc., 81, 2953-2962, 2000.

Dowell, D. C. and Shapiro, A.: Stability of an Iterative DualDoppler Wind Synthesis in Cartesian Coordinates, J. Atmos. Oceanic Technol., 20, 1552-1559, 2003.
Goh, Y. K. and Holt, A. R.: Analysis of Three - Dimensional Wind Fields from Two Operational Doppler Radars, in: Proceeding of European Conference on Radar in Meteorology and Hydrology (ERAD), 2, 35-40, Copernicus GmbH, Germany, 2004.

Haase, G. and Landelius, T.: Dealiasing of Doppler radar velocities using a Torus Mapping, J. Atmos. Ocean. Technol., 21, 15661573, 2004.

Laroche, S. and Zawadzki, I.: A variational analysis method for retrieval of three-dimensional wind field from single-Doppler radar data, J. Atoms. Sci., 51, 2664-2682, 1994.

Lhermitte, R. M.: Dual-Doppler radar observations of convective storm circulation, in: Preprint, 14th Conf. on Radar Meteorology, 153-156, Amer. Meteor. Soc., 1970.

Lindskog, M., Salonen, K., Järvinen, H., and Michelson, D. B.: Doppler radar wind assimilation with HIRLAM 3DVAR, Mon. Wea. Rev., 132, 1081-1092, 2004.

Shapiro, A., Ellis, S., and Shaw, J.: Single-Doppler retrievals with Phoenix II data: Clear air and microburst wind retrievals in the planetary boundary layer, J. Atoms. Sci., 44, 2640-2656, 1995.

Watson, R. J.: Data Comparisons for Spatially Separated Meteorological Radars, Phd thesis, Department of Electronic Systems Engineering, University of Essex, 1996.

Wüest, M., Schmid, W., and Zawadzki, I.: Improving singleDoppler wind retrievals with secondary wind field data, in: Preprint 29th Int. Conf. on Radar Met., Amer. Meteor. Soc., 138$141,1999$. 